\title{
Thematisierung von „Religion" in der österreichischen Politikwissenschaft. Status Quo, Tendenzen und kritische Anmerkungen
}

\author{
Farid Hafez
}

This article examines the relevance of religion in the Austrian political science-landscape. Based on research conducted by political scientists Kenneth D. Wald \& Clyde Wilcox (2006) and Steven Kettell (2012), who see an underexposed position of religion in USpolitical science, this article raises the question of the relevance of religion in the Journal of Austrian Political Science. A content analysis will be conducted for all volumes published between 2000 and 2015. This analysis will be compared to findings from an analysis of the Austrian Journal of Sociology. Findings show that compared to the USA, as well as to the Austrian Journal of Sociology, religion is addressed significantly more often in the Journal of Austrian Political Science. This is explained by: 1. especially engaged political scientists in the research on religion, and: 2 . a comparably small size of political science landscape in Austria.

Farid Hafez is researcher at the Department of Political Science at the University of Salzburg. Hafez earned his Ph.D. in Political Science at the University of Vienna. He has had research stays in the USA as Fulbright professor (UC Berkeley), teaching residencies in Indonesia, Turkey and the USA. Since 2010 he has been the editor of the bilingual Islamophobia Studies Yearbook, since 2016 co-editor of the European Islamophobia Report. In 2009 he was, together with John Bunzl, awarded with the Bruno-KreiskyAward for the Political Book of the Year. Most recent publication as co-editor: "Jung, muslimisch, österreichisch" (New Academic Press, 2016).

\section{Einleitung}

„Nun sag, wie hast du's mit der Religion? Du bist ein herzlich guter Mann, allein ich glaub, du hältst nicht viel davon. “1 Die Gretchenfrage aus Johann Wolfgang von Goethes Faust I ließe sich meinem Erachten nach ebenso auf die Politikwissenschaften beziehen. Die hier vorgelegte Analyse beabsichtigt, eine kritische Selbstreflexion der Politikwissenschaft voranzutreiben, indem der Frage nach der Bedeutung von Religion in der Politikwissenschaft nachgegangen wird. Darauf aufbauend werden Potentiale und Risiken einer Beschäftigung mit diesem Themengebiet, nicht zuletzt im Zusammenhang mit Migration, andiskutiert. Die

1 Goethe 1899, Vers 3415. 
beiden deutschen Politikwissenschaftler Ulrich Willens und Michael Minkenbergs meinten in einer Sonderausgabe der deutschen Politischen Vierteljahresschrift aus dem Jahr 2002, dass die Beschäftigung mit „Religion“ in ihren unterschiedlichsten Ausprägungen in der ,deutschen bzw. europäischen politikwissenschaftlichen Auseinandersetzung bislang eher ein Schattendasein [...] im Gegensatz zur Geschichtswissenschaft, zur Soziologie und zur Rechtswissenschaft" ${ }^{\prime 2}$ geführt habe. Dieser Befund ist in der Disziplin ein gemeinhin oft geteilter, der auch empirisch belegt ist, wie die weiter unten dargestellten Beiträge von Wald \& Wilcox sowie Kettell veranschaulichen.

Dieser Beitrag macht es sich zur Aufgabe, auf Basis der von Wald / Wilcox sowie Kettell empirisch erhobenen Daten und formulierten Hypothesen die österreichische Politikwissenschaft auf ihre Bedeutung von Religion hin zu befragen.

Die zentrale Forschungsfrage lautet: Wie wird Religion in den Publikationen der österreichischen Politikwissenschaft behandelt? Dazu wurden 403 Artikel der Österreichischen Zeitschrift für Politikwissenschaft aus den Jahren 2000 bis 2015, auf die Frage hin untersucht, inwiefern Religion ein Thema politikwissenschaftlicher Reflexion darstellte. Die Artikel wurden einer Inhaltsanalyse unterzogen. In einem ersten Schritt wurden der Titel und die Zusammenfassungen untersucht. In einem zweiten Schritt wurde nach den aneinandergereihten Buchstaben „religi*“ gesucht, um alle möglichen Abwandlungsformen wie „Religion“, „religiös“ oder ,religions-“ zu beinhalten. Die Inhaltsanalyse wurde durch zwei voneinander unabhängige Personen vorgenommen, um anschließend nach einem Vergleich diskutiert zu werden.

Da Österreich ein kleines Land mit einer überschaubaren politikwissenschaftlichen Institutionslandschaft ist, stellt sich keine Problematik der Frage nach der politikwissenschaftlichen Vorzeigezeitschrift. Die Österreichische Zeitschrift für Politikwissenschaft (ÖZP) ist das einzige politikwissenschaftliche Journal, welches in Österreich existiert. Damit bildet die ÖZP die politikwissenschaftliche Forschung am ehesten ab. Sie wurde 1972, ein Jahr nach der Verankerung je eines politikwissenschaftlichen Instituts in Salzburg und Wien (die ersten beiden in Österreich), gegründet. Heute wird sie von der Österreichischen Gesellschaft für Politikwissenschaft ${ }^{3}$ herausgegeben, bei der im Jahre 2015 über 600 AkademikerInnen der einzelnen Institute und Abteilungen, an denen Politikwissenschaft gelehrt wird, individuelle Mitgliedschaften aufweisen. ${ }^{4}$ Es gibt freilich auch weitere qualitativ hochwertige österreichische Periodika. Jedoch sind diese deutlich anders geartet: Dazu zählen etwa die seit 1974 erscheinende Europäische Rundschau oder International. Die Zeitschrift für Internationale Politik, die kein double blind peer review-Verfahren anwenden. Zudem gibt es

2 Willems / Minkenberg 2003.

3 Vgl. Internetquellen: ÖZP.

4 Vgl. Internetquellen: Österreichische Gesellschaft für Politikwissenschaft. 
Zeitschriften mit staatlich-institutionellem Naheverhältnis wie das bis zum Jahr 1808 zurückgehende Fachperiodikum des Verteidigungsministeriums Österreichische Militärische Zeitschrift oder das 1979 erstmals erschienene Jahrbuch für internationale Sicherheitspolitik, das ebenso dem Bundesheer zuzurechnen ist. Das von der Politischen Akademie (ÖVP) seit 1977 herausgegebene und mehrheitlich von politischen PraktikerInnen aller Couleur verfasste Österreichische Jahrbuch für Politik gilt als ein wichtiges politisches bis politikwissenschaftliches Periodikum. Jedoch sind diese Zeitschriften nicht nach Akademischen Kriterien begutachtet. Zudem publizieren in ihnen auch viele Nicht-PolitikwissenschaftlerInnen. Nur wenige außeruniversitäre akademische Einrichtungen weisen in Österreich Periodika auf. So wurde das Österreichische Jahrbuch für Internationale Politik (1984-2002), welches die Österreichische Zeitschrift für Außenpolitik (1960-1983) beerbt hatte, im Jahre 2002 eingestellt. Erst seit 2001 betreibt das Österreichische Institut für Internationale Politik das begutachtete Jahrbuch ADD-ON Jahrbuch oiip: Wiener Beiträge zur Internationalen Politik. Wenige Periodika weisen ein doppeltes, blindes Begutachtungsverfahren auf und sind gleichzeitig etwas älter. Das 1986 gegründete Journal of Development Studies ${ }^{5}$ und das 1997 erstmals erschienene European Integration online Papers (EIoP) ${ }^{6}$ sind zwei der wenigen Ausnahmen, die beide aber gleichzeitig sehr spezifisch in ihrer thematischen Ausrichtung sind. Die ÖZP sticht hiermit als breit angelegte und gleichzeitig spezifisch politikwissenschaftliche Zeitschrift heraus, weshalb es wenig Sinn macht, diese thematisch sehr engen und zudem nicht vierteljährlich, sondern oftmals nur jährlich erscheinenden Periodika mit in das Sample hineinzunehmen.

Von allen Beiträgen der ÖZP zwischen 2000 und 2015 lagen digitale Versionen vor, die die automatische Kodierung erleichterten. Die Publikationen wurden entsprechend der Zentralität religiöser Themengebiete kodiert. Als primäre Begriffe wurden jene identifiziert, wo der Begriff des Religiösen oder ein inhaltlich religiöses Thema (Buddhisten, Christen, Muslime etc.) behandelt wurde (im Titel oder Abstract enthalten) bzw. Religion als eine zentrale Variable behandelt wurde. Als sekundäre Artikel wurden jene genommen, in denen Religion als ein Faktor unter mehreren behandelt wurde und eher als Nebenthema Erwähnung fand.

Auch wenn diese Analyse einen Einblick in eine gesamtpolitikwissenschaftliche Tendenz gibt, kann sie gleichzeitig nicht als vollständig repräsentativ für alle PolitikwissenschaftlerInnen gelten, da weitere Publikationen österreichischer PolitikwissenschaftlerInnen mit Bezug auf Religion in spezifischeren internationalen Journalen erscheinen. ${ }^{7}$ Dennoch lässt sie eine Aussage zu, welche Ten-

\footnotetext{
5 Vgl. Internetquellen: Mattersburger Kreis.

6 Vgl. Internetquellen: European Integration online Papers.

7 Vgl. beispielhaft Internetquellen: Publikationsliste der Wiener Politikwissenschaftlerin Sieglinde Rosenberger.
} 
denz es im Zusammenhang mit der Bedeutung von Religion in der österreichischen Politikwissenschaft gibt. Im Falle der ÖZP gilt, dass sie nicht nur politikwissenschaftliche, sondern im weitesten Sinne sozialwissenschaftliche Artikel veröffentlicht. Dennoch reflektiert die Hereinnahme soziologischer, anthropologischer etc. Beiträge in die ÖZP zur Thematik von Religion die Bedeutung, die diese dem Faktor Religion beimisst.

\section{Religion in der US-amerikanischen politikwissenschaftlichen Forschung}

Auch wenn Autoren wie José Casanova festhalten, dass Europa ein Ausnahmekontinent sei, der sich wie kein anderer Kontinent der Säkularisierungsthese im Sinne einer Zurückdrängung von Religion im öffentlichen Raum verschrieben hätte, ${ }^{8}$ so erfahren Debatten um eine postsäkulare Ära, in der Religion auch als legitime Ressource von Politik gehandelt werden kann, ${ }^{9}$ eine fortschreitende Popularisierung. ${ }^{10}$ Im Gegensatz dazu sind die Vereinigten Staaten von Amerika ein Land mit signifikanter Präsenz des Religiösen in der öffentlichen und politischen Sphäre..1 Das reicht von der Bedeutung von Religion in der schwarzen Bürgerrechtsbewegung ${ }^{12}$ bis hin zur Wahlkampfauseinandersetzung zwischen Republikanern und Demokraten, wenn z. B. über Obama behauptet wird, er sei Muslim. ${ }^{13}$ Paradoxerweise scheint aber auch in den USA das Säkularisierungsparadigma in der Politikwissenschaft Fuß gefasst zu haben. So teilen auch USamerikanische PolitikwissenschaftlerInnen den Befund des Schattendaseins der Religion in den Politikwissenschaften und nahmen diesen zum Anlass für Forschungen. 2006 veröffentlichten Kenneth D. Wald und Clyde Wilcox einen Artikel, in dem sie spezifischer der Frage auf den Grund zu gehen versuchten, warum in der führenden Zeitschrift der globalen Politikwissenschaft, der American Political Science Review, der Faktor Religion eine, wenn überhaupt, marginale Rolle einnimmt. ${ }^{14}$ Entgegen der Annahme, dass die Unterrepräsentation von Religion auf eine antireligiöse Befangenheit der Politikwissenschaften im Besonderen zurückzuführen sei, machen die beiden Autoren vier Faktoren für diesen Befund verantwortlich:

\footnotetext{
8 Vgl. Casanova 2013.

9 Vgl. Habermas / Ratzinger 2005.

10 Vgl. beispielhaft: Barbato 2011.

11 Vgl. Brocker 2013.

12 Vgl. Morris 2014.

13 Vgl. Hafez 2013.

14 Vgl. Wald / Wilcox 2006.
} 
1. die intellektuellen Grundlagen der Disziplin;

2. den sozialen Hintergrund der Proponenten;

3. die Komplexität der Bewertung von „Religion“;

4. die ereignisgesteuerte Agenda der Disziplin.

Ad 1: Mit den intellektuellen Grundlagen der Disziplin meinen Wald und Wilcox den bei Kernthemen anzufindenden frühen Fokus der Politikwissenschaft auf nicht-religiöse Faktoren wie etwa Klasse als erklärende Variable. So betrachtet Lijphart religiöse Werte lediglich als Begleiterscheinung, ohne diesen größeres Erklärungspotential zuzuschreiben. Anders als die Soziologie, in der, angeregt durch Marx, Weber und Durkheim, der Einfluss des Religiösen in Gesellschaft und Politik untersucht wurde, habe die Politikwissenschaft ihr Angebot abhängiger Variablen stärker limitiert. In der Politikwissenschaft hätte dementsprechend weder in den frühen Jahren des Reviews im Rahmen des Institutionalismus, noch in der darauffolgenden Phase, die durch den empiristischen-behavioristischen Ansatz geprägt war, Religion große Aufmerksamkeit geschenkt. Zudem habe die Verpflichtung US-amerikanischer PolitikwissenschaftlerInnen zu liberal-demokratischen Werten diese davon abschrecken lassen, einen Blick auf institutionalisierte Religion zu wagen oder diese gar als konstitutiven Bestandteil demokratischer Ordnungen zu betrachten. Stattdessen wurde Religion als zu zähmende Kraft gedeutet, die keiner weiteren Beachtung mehr notwendig schien. Die Dominanz der Säkularisierungsthese als Teil der Modernisierungsthese ${ }^{15}$ steht auch nach Wald / Wilcox für diese distanzierte Haltung. ${ }^{16}$

15 Im Zusammenhang damit ist auch der Teilbereich der Politischen Theorie gefragt, in deren Bereich ich das Säkularisierungstheorem verorten würde. Mit der Säkularisierungsthese wird zum einen beschrieben, dass auf ,struktureller und institutioneller Ebene eine Autonomisierung und Differenzierung von Politik und (christlicher) Religion“ (Willems / Minkenberg 2003, S. 18) geschieht, auf ,politisch-kultureller Ebene eine Erosion organisierter (christlicher) Religion“ eintritt und weiters auf gesellschaftlicher Ebene ein „Rückzug verbliebener organisierter Religion sowie religiöser Symbole und Praktiken aus der Sphäre der Politik“ (Ebd., S. 19) stattfindet, der die Religion in den nicht-politischen Bereich des Privaten verbannt. Dieses Säkularisierungstheorem ist trotz seiner gesellschaftlichen Wirkmächtigkeit im Sinne einer Idee bereits früh bestritten worden, wie die Begriffe „politische Theologie“, „politische Religion“ (siehe dazu: Ley / Neisser / Weiss 2003) oder „Zivilreligion“ zeigen. Umso mehr müsste eine Beschäftigung mit „zugewanderten Religionen“, die nicht die politisch-historische Entwicklung des europäischen Christentums teilen, dazu anregen dieses ohnehin von vielen in Frage gestellte Theorem zu überdenken. Nach Wald / Wilcox habe die Verpflichtung US-amerikanischer PolitikwissenschaftlerInnen zu liberal-demokratischen Werten diese davon abgehalten, einen Blick auf institutionalisierte Religion zu wagen oder diese gar als konstitutiven Bestandteil demokratischer Ordnungen zu betrachten. Das Böckenförde-Paradoxon, wonach der ,freiheitliche, säkularisierte Staat [...] von Voraussetzungen, die er selbst nicht garantieren kann“ (Böckenförde 1992, S. 112) lebt, ebenso wie 
Ad 2: Was den sozialen Hintergrund der Gruppe der PolitikwissenschaftlerInnen betrifft, so meinen Wald / Wilcox, dass zwar keine Identifikation mit Religion für eine Beschäftigung mit dieser notwendig sei, hingegen eine ,geringe Vertrautheit mit Religion zu einer geringeren Beschäftigung damit führen“"17 würde. Sie ziehen ihre Schlüsse basierend auf einer Vielzahl an Studien über das persönliche Verhältnis von PolitikwissenschaftlerInnen zu Religion. Die Befunde dieser Studien legen nahe, dass die Gruppe der PolitikwissenschaftlerInnen sehr wenige religiöse und anti-religiöse Einstellungen aufweist. Vielmehr scheint eine Mehrzahl der WissenschaftlerInnen der Religion indifferent gegenüberzustehen. Daraus schließen die Autoren, dass Religion als erklärende Variable meist aus dem Blickfeld der Analyse fällt.

Dies lässt sich am Begriff der Post-Säkularität veranschaulichen. Mit dem Einzug des Begriffs der Post-Säkularität in die Sozialwissenschaften ließe sich provokant die Frage stellen, ob die Markierung dieser Ära ohne Datum, in der sich angeblich eine Rückkehr des Religiösen abzeichnet, nicht eher den Blick eines wie oben beschriebenen Milieus der religiös indifferenten Personen reflektiert. Beispielhaft sei die sogenannte Rückkehr der Christlichen Rechten auf die politische Bühne angeführt. Die Fundamente dieser Bewegung wurden bereits zu Beginn des 20. Jahrhunderts gelegt. ${ }^{18}$ Dies deutet demnach darauf hin, dass diese Bewegung - wenn sie auch jahrzehntelang keine parteipolitischen und öffentlichen Ansprüche stellte - sich über Jahrzehnte abseits des Bewusstseins eines Gros der PolitikwissenschaftlerInnen entwickeln konnte. Sie ist damit schon lange da gewesen, aber eben erst mit ihrer Unübersehbarkeit auch in das Blickfeld der Politikwissenschaften gerückt. Ist die jahrzehntelange Blindheit diesem Milieu gegenüber damit nicht schlussendlich der Blindheit des Milieus eines Gros der PolitikwissenschaftlerInnen geschuldet?

Ad 3: Die Komplexität des Religiösen als zu erfassendem Gegenstand, den es zu messen gilt, ist für Wald / Wilcox ein weiterer Grund für die geringe Beschäftigung mit Religion in den Politikwissenschaften. ${ }^{19}$ Überspitzt könnte man sagen, seien „religiöse Analphabeten“ der Komplexität des Gegenstandes „Religion“ nicht gewachsen. Darüber hinaus ist zu beobachten, dass oftmals nicht Politik-

Habermas' Ansatz, Religion als Quelle deliberativer Kräfte für die Demokratie zu inkludieren, ermöglicht auch im Zusammenhang mit Migration eine über das Christentum hinausgehende Diskussion anzuregen, die bisher nur in Ansätzen geführt wurde. Damit erschließt sich für die Politikwissenschaft als Disziplin das Potential, einen lange währenden Tunnelblick zu durchbrechen. Einzelne Persönlichkeiten in benachbarten Disziplinen tun dies ohnehin schon länger. Beispielhaft für die Geschichtswissenschaften: Lehmann 1997 und Jakubowski-Tiessen 2004.

16 Vgl. Wald / Wilcox, S. 525 f.

17 Ebd., S. 526.

18 Vgl. Ostendorf 1998.

19 Vgl. ebd., S. $526 \mathrm{f}$. 
wissenschaftlerInnen Beiträge zu politischem Denken und Religion verfassen, sondern ReligionswissenschaftlerInnen. Nehmen wir als ein Beispiel die kürzlich erschienene Princeton Encyclopedia of Islamic Political Thought ${ }^{20}$, die von einem führenden Islamwissenschaftler herausgegeben wurde. Hier stehen die PolitikwissenschaftlerInnen eindeutig in der zweiten Reihe. Keine/r der HerausgeberInnen gehört zur Disziplin der Politikwissenschaft. Für Österreich gilt: Die ÖGPW hat Tagungen zu Religion gemacht. ${ }^{21}$ Bezeichnend ist aber auch hier, dass eine Praktische Theologin wie Regina Polak im Rahmen der ÖGPW-Tagung ein Panel zu Religion, Migration und Inklusion anbietet. ${ }^{22}$ Während Willems und Minkenberg bereits 2002 anmerkten, dass ,angesichts der Vielzahl von Konflikten und Regelungsgegenständen sowie der spezifischen Logik und Dynamik darauf bezogener politischer Prozesse [...] es inzwischen durchaus Sinn (macht, Autor), von einem eigenständigen Politikfeld ,Religionspolitik' zu sprechen“23, gleichzeitig auch eine Vielzahl an Studien in diesem Bereich in den letzten Jahren erschienen ist, gilt es festzuhalten, dass institutionell diese Einsicht in Österreich nur rudimentär abgebildet wird. Wahlmodule zum Themenspektrum des Religiösen gibt es in keinem einzigen Curriculum eines österreichischen Politikwissenschaftsinstituts. Vereinzelt werden zwar Lehrveranstaltungen zur Religionspolitik basierend auf jüngeren Forschungen angeboten, wie etwa im Falle der Politikwissenschaftlerin Kristina Stoeck $1^{24}$, von einer Institutionalisierung ist man aber noch weit entfernt.

Ad 4: Nun zum letzten von Wald und Wilcox angeführten Punkt. Während die Wahl des Katholiken Kennedy zum Präsidenten der Vereinigten Staaten US-amerikanische PolitikwissenschaftlerInnen glauben ließ, dass das anti-religiöse Vorurteil (in diesem Fall gegen KatholikInnen) ${ }^{25}$ ausgestorben sei, wurden mit der islamischen Revolution im Iran 1979 und dem Aufstieg der Christlichen Rechten in den USA zum einen die Weltöffentlichkeit, zum anderen die Vereinigten Staaten an die bedeutende Rolle der Religion erinnert.

\section{Bulliet 2012.}

21 Vgl. Internetquellen: Österreichische Gesellschaft für Politikwissenschaft: Tagung: Europa und das Andere - Konflikte um Geschlecht und Religion.

22 Vgl. Internetquellen: Österreichische Gesellschaft für Politikwissenschaft: Call for Papers.

23 Willems / Minkenberg 2003, S. 16.

24 Vgl. Internetquellen: Stoeckl, Teaching. So ist das von den beiden deutschen PolitikwissenschaftlerInnen Heidrun Abromeit und Wewer Göttrik 1989 veröffentlichte Buch zum Verhältnis von Kirche und Politik im Jahre 2013 wieder aufgelegt worden. Erst kurz vor Entstehen dieses Artikels hat der deutsche Politikwissenschaftler ein aktuelles Werk zum Verhältnis von Politik und Religionsgemeinschaften veröffentlicht. Diese beiden Monographien zeigen das Defizit der Politikwissenschaft im Feld der Religionspolitik auf. Siehe dazu: Abromeit / Wewer 2013 sowie Beyme 2015.

25 Vgl. Carty 2004. 
Auch wenn die beiden Autoren angeben, dass seither die Beschäftigung mit Religion gewachsen sei, wie z. B. die Integration von Religion in verschiedenen Projekten (hier sind u. a. die American National Election Studies (ANES), das Pew Center, die World Values Study und der Eurobarometer zu nennen), veranschaulichen, ${ }^{26}$ laufe die Bedeutung von Religion in der Politikwissenschaft dennoch Gefahr, ebenso schnell wie die Beschäftigung mit ihr einen Anstieg erfuhr auch wieder zu verschwinden. Jene PolitikwissenschaftlerInnen nämlich, die Forschungen zu Religion aus politikwissenschaftlicher Perspektive durchgeführt haben, stünden von manchen ihrer KollegInnen in der Kritik, dass ihre Beiträge kaum zu einem Mehrwert für die Disziplin als solche beigetragen hätten. Dies impliziere, dass die Beschäftigung mit Religion nur für jene von Interesse sei, die sich selbst als religiös definieren oder sich für Religion im Spezifischen interessieren würden. ${ }^{27}$ Daran anknüpfend kann die Frage gestellt werden, welche Rolle Religion zur Erklärung zentraler Begriffe in den Politikwissenschaften wie Macht, Konflikt, Herrschaft, Ordnung, Gesellschaft, Partizipation, Staat, Verfassung, Gesetz, Partei, Parlament, Regierung, Demokratie, Wahlen, Souveränität oder Ritual beiträgt. Eine systematische Abarbeitung dieser Begriffe in Verbindung mit Religion ist aus politikwissenschaftlicher Sicht meinem Wissen nach ausständig. ${ }^{28}$

Ein kurzer Blick auf das Standard-Einführungswerk der österreichischen Politikwissenschaft - das Handbuch Politik in Österreich ${ }^{29}$ - offenbart, dass auch hier Religion eine mehr als untergeordnete Rolle spielt. Der darin von Barbara Prainsack enthaltene Beitrag zu Religion und Politik in Österreich ${ }^{30}$ veranschaulicht, dass die Rolle von Religion im Wesentlichen auf der Rezeption rechtswissenschaftlicher Arbeiten basiert (insbesondere zum Staat-Kirche-Verhältnis) und in der Politikwissenschaft bis zum Jahre 2006 keine genuine Arbeit sichtbar war. Dieser Befund, dass die Rolle von Religion tendenziell auf das Staat-Kirche-Verhältnis reduziert wird, ist kein genuin österreichischer. Das zeigt die idente Feststellung von Wald / Wilcox für die USA. ${ }^{31}$

Wald und Wilcox sind nicht die einzigen, die sich der Frage der Thematisierung von Religion in der Politikwissenschaft gewidmet haben. Knapp zehn Jahre nach Willens und Minkenbergs Aussage über das Schattendasein der Religion in der Politikwissenschaft veröffentlichte Steven Kettell eine quantitative Auswertung, in der er der Annahme nachging, dass die Politikwissenschaften Religion weitgehend ignorieren würden. Die Validität dieser Aussage überprüfte er, indem er

\footnotetext{
26 Vgl. Willems / Minkenberg 2003, S. 527.

27 Vgl. ebd., S. 528.

28 Das bedeutet nicht, dass nicht zu manchen dieser Bereiche gearbeitet wurde. Siehe etwa die Vielzahl an Arbeiten des deutschen Politikwissenschaftlers Hans Maier.

29 Dachs 2006.

30 Prainsack 2006.

31 Vgl. Wald / Wilcox 2006, S. 525.
} 
die - gemessen am ISI Impact-Faktor - 20 höchst gereihten Journale aus dem Bereich der Politikwissenschaft und der Soziologie in den Jahren 2000 bis 2010 auf die Thematisierung von „Religion“ hin befragte. ${ }^{32}$ Zehn Jahre nach der Einschätzung von Willens und Minkenberg für Deutschland und Europa wurde also die Situation auf globaler Ebene in den Blick genommen.

Grundsätzlich konnte Kettell die These bestätigen. Interessant ist das Ergebnis der Auswertung der Frage, in welchen Themengebieten „Religion“ untersucht wurde. Auffallend ist, womit die Thematisierung von Religion in politikwissenschaftlichen Untersuchungen verknüpft ist. Ganz oben rangieren die Themenkomplexe „Gewalt, Konflikt, Terrorismus“ (21,7 \%), „Islam und MuslimInnen“ $(20,6 \%)$ sowie „US-amerikanische Politik und Gesellschaft“ (17,5 \%), gefolgt von „Zivilgesellschaft“ und „Wahlen“. Was hierbei auffällt, ist ein Übermaß an Themen, die auf die ereignisgesteuerte Agenda der Disziplin im Sinne von Wald / Wilcox hinweisen. Die Verteilung der Themen reflektiert außerdem, welche dieser Themen AutorInnen wichtig erschienen und welche nicht. Wie der Anthropologe Matti Bunzl erklärt, ist es mitunter auch eine politische Frage, und es ließe sich in der Umkehrung sagen: nicht ausschließlich eine erkenntnistheoretisch-analytische Frage, was wie thematisiert wird und worüber geschwiegen wird. ${ }^{33}$

\begin{tabular}{|l|l|l|}
\hline \multicolumn{3}{|l|}{ Primary Coded Articles by Main Themes } \\
\hline & Theme & $\%$ \\
\hline 1 & Violence, conflict, and terrorism & 21,7 \\
\hline 2 & Islam / Muslims & 20,6 \\
\hline 3 & U.S. politics and society & 17,5 \\
\hline 4 & Social capital / civic engagement & 10,3 \\
\hline 5 & Elections and voting & 10,3 \\
\hline 6 & Democracy & 7,2 \\
\hline 7 & Political science & 6,2 \\
\hline 8 & Nationalism & 5,2 \\
\hline 9 & Women/gender & 4,1 \\
\hline 10 & Life and death issues & 3,1 \\
\hline
\end{tabular}

Quelle: Table 2, Steven Kettell (2012)

32 Vgl. Kettell 2012.

33 Vgl. Bangstad / Bunzl 2010, S. 215. 
Gleichzeitig muss aber auch in Rechnung gestellt werden, dass einiges im Verhältnis von Politikwissenschaft und Religion in Bewegung gekommen ist. Mit dem Jahr 2007 wurde von der Sektion „Religion and Politics“ der APSA (American Political Science Association) ein Journal mit dem Namen Politics and Religion gegründet, um der Unterbeleuchtung dieses Themas etwas entgegenzuhalten. Vergleichbares gibt es im deutschsprachigen Raum nicht.

\section{Religion in der ÖZP}

Insgesamt bilden 403 Artikel in der ÖZP den Forschungscorpus. Exkludiert wurden die in der ÖZP erschienenen Buchrezensionen. Im Gegensatz zu der Untersuchung bei Kettell wurden bei der hier vorliegenden Analyse ein größerer Zeitraum und gleichzeitig eine geringere Anzahl an Publikationen in die Analyse mithineingenommen. Insgesamt beschäftigten sich 33 der 403 Artikel, das entspricht 8,18 \% primär mit dem Begriff der „Religion“ Hierzu zählen Artikel, die sich mit dem politischen Islam, kulturellen Differenzen, politischer Theorie, aber auch Imaginationen des „Anderen“ im Sinne von Antisemitismus, Anti-Ziganismus und Islamfeindlichkeit auseinandersetzen.

22,58 \% (insgesamt 91 Beiträge) setzen sich sekundär mit diesem Gegenstand auseinander. Damit sind Artikel gemeint, die am Rande den Gegenstand der Religion andiskutieren, ohne diesen als zentrale Variable in ihrem Artikel zu thematisieren.

Damit ist der Anteil an Beiträgen zum Thema „Religion“ im Vergleich zu den US-amerikanischen politikwissenschaftlichen Journalen signifikant höher. Während in den führenden US-amerikanischen politikwissenschaftlichen Journalen der Anteil an primär kodierten Artikeln bei 1,34 \% liegt und insgesamt bei $2,54 \%$, ist jener des ÖZP insgesamt bei 30,76 \% und im Falle der primär kodierten mit 8,18 \% fast drei Mal so hoch wie der Wert US-amerikanischer Fachzeitschriften. Die führende American Political Science Review liegt nahe dem von Kettell gemessenen Durchschnitt.

\begin{tabular}{|l|l|l|}
\hline Zeitschriftenname & Primär kodiert (\%) & Sekundär kodiert (\%) \\
\hline $\begin{array}{l}\text { Österreichische Zeitschrift für } \\
\text { Politikwissenschaft }\end{array}$ & 8,18 & 30,76 \\
\hline American Political Science Review & 2,17 & 2,61 \\
\hline $\begin{array}{l}\text { US-amerikanische Zeitschriften } \\
\text { (20 höchst gereihten) }\end{array}$ & 2,54 & 1,34 \\
\hline
\end{tabular}

Quelle: Darstellung des Autors 
Nehmen wir das Themenspektrum, welches innerhalb der primär kodierten Artikel behandelt wurde, genauer in den Blick, so zeigt sich folgendes Bild:

\begin{tabular}{|l|l|l|}
\hline \multicolumn{2}{|l|}{ Primär kodierte Artikel nach Hauptthemen } \\
\hline & Thema & $\%$ \\
\hline 1 & Islam & 35,5 \\
\hline 2 & Politische Theorie & 20,0 \\
\hline 3 & Cleavage-Theorie & 13,3 \\
\hline 4 & Antisemitismus & 9,7 \\
\hline 5 & Rechtspopulismus & 9,7 \\
\hline 6 & Religionszugehörigkeit & 6,5 \\
\hline
\end{tabular}

Quelle: Darstellung des Autors

Mehr als ein Drittel, 35,5 \% der Beiträge, thematisieren den Islam als Religion. Diese setzen sich aus Artikel zum Islam im Allgemeinen (29\%) und dem politischen Islam als politischem Akteur (6,6 \%) auseinander. Es ließen sich noch weitere Unterteilungen in Islambilder und Religionspolitik machen. Gleichzeitig wäre es möglich, manche dieser Islam-Themen mit anderen Themen zusammenzuführen. So wird „Islam“ nicht explizit im Zusammenhang mit der Cleavage-Theorie behandelt, durchaus aber im Zusammenhang mit Rechtspopulismus. Das ist insofern aber zweitrangig, als das Islam-Thema insgesamt vermutlich auf die ereignisgesteuerte Agenda der Politikwissenschaft hindeutet. Gleichzeitig ist zu beachten, dass die ÖZP v. a. vor 2009 ausschließlich Themenhefte und keine offenen Hefte veröffentlichte. Indem etwa Bd. 37, Nr. 4 (2008) dem Thema „Islam im öffentlichen Raum. Debatten und Regulationen in Europa“ gewidmet war, und dabei gleich sieben Artikel zum Themengebiet Islam aufweist, würde die ÖZP ohne diesem Heft nur zwei Artikel mit Islam-Thema enthalten, was bereits die Häufigkeit mit Blick auf alle Ausgaben relativiert. Des Weiteren ist anzumerken, dass die beiden Herausgeberinnen dieser Ausgabe, die Wiener Politikwissenschaftlerinnen Sieglinde Rosenberger und Birgit Sauer, zeitgleich eine Vielzahl an Arbeiten zu diesem Themenkomplex - besonders international veröffentlicht haben und insofern das Themenheft auch zentrale Forschungsschwerpunkte zweier Universitätsprofessorinnen im Bereich der Politikwissenschaften widerspiegelt. ${ }^{34}$

34 Siehe dazu beispielhaft: Rosenberger / Sauer 2012 sowie Rosenberger / Sauer 2013 . 


\begin{tabular}{|l|l|l|l|l|}
\hline \multicolumn{6}{|l|}{ Primär kodierte Artikel: Vergleich zwischen USA und ÖZP } \\
\hline & US-Journale & $\%$ & ÖZP & $\%$ \\
\hline & Thema & 21,7 & Islam & 35,5 \\
\hline 1 & Terror, Gewalt, Konflikt & 20,6 & Politische Theorie & 20,0 \\
\hline 2 & Islam/Muslime & 17,5 & Cleavage-Theorie & 13,3 \\
\hline 3 & US-Politik und Gesellschaft & 10,3 & Antisemitismus & 9,7 \\
\hline 4 & Sozialkapital & 10,3 & Rechtspopulismus & 9,7 \\
\hline 5 & Wahlen & 7,2 & Religionszugehörigkeit & 6,5 \\
\hline 6 & Demokratie & & & \\
\hline
\end{tabular}

Quelle: Table 2, Steven Kettell (2012) und Darstellung des Autors

Im Vergleich zu den USA zeigt sich, dass die Themengebiete Terror, Gewalt und Konflikt gar nicht im Zusammenhang mit Religion aufscheinen. Einzig bezüglich des Themenfelds Islam/Muslime besteht eine Ähnlichkeit darin, dass es sowohl in den US-amerikanischen Journalen wie auch in der ÖZP im Bereich der obersten beiden Themen rangiert. Die Beschäftigung mit dem Antisemitismus ist weiters dem Umstand geschuldet, dass auch in diesem Fall ein Themenheft mit dem Schwerpunkt „Antisemitismus und die Transformation des Nationalen“ (Bd. 39, Nr. 4, 2010) herausgegeben wurde. Hier könnte der Gesichtspunkt aufgeworfen werden, dass im Falle einer Existenz von spezialisierten politikwissenschaftlichen Fachzeitschriften zum Themenkomplex Religion diese Themenhefte dort besser untergebracht gewesen wären. Das ist insbesondere für den US-amerikanischen Raum herauszustreichen. Die bereits erwähnte Zeitschrift Politics and Religion der Sektion „Religion and Politics“ der APSA fängt die Spezialisierung auf das Themengebiet Religion zu einem gewissen Grad auch auf. Sie gibt an, in der Zwischenzeit beinahe 100 Einreichungen im Jahr zur Begutachtung zu erhalten. ${ }^{35}$ Somit ist der Vergleich zwischen der ÖZP und den US-amerikanischen Journalen stärker zu problematisieren, da es im deutschsprachigen Raum und insbesondere in Österreich nichts Vergleichbares gibt. PolitikwissenschaftlerInnen sind im österreichischen Kontext quasi gezwungen, sich auf das einzige peer-reviewed Journal - die ÖZP - zu konzentrieren. Gleichzeitig ist wiederholt zu betonen, dass etwa Rosenberger und Sauer mit weiteren ForscherInnen eine Fülle an Artikeln zum Themenkomplex Religion und Politik in internationalen Journalen veröffentlicht haben, was sich in der ÖZP nicht widerspiegelt.

Der Vergleich mit den USA ist erhellend und problembehaftet zugleich. Zwar sind die Werte im Vergleich zu den USA signifikant höher. Die Unterscheidung nach

Vgl. Internetquellen: Politics and Religion. 
primärer und sekundärer Kodierung ist objektiv nachvollziehbarer und weniger subjektiv als die Zuteilung zu Themen. Dennoch zeigt dies eine Tendenz innerhalb der Disziplin auf. ${ }^{36}$ Das gilt für die ÖZP insofern besonders, weil es sich um das politikwissenschaftliche Journal handelt. Gleichzeitig macht es aber aus einer innerösterreichischen Perspektive auch Sinn, verwandte Disziplinen zum Vergleich heranzuziehen und somit das Ergebnis in einen österreichischen sozialwissenschaftlichen Kontext zu stellen. Aus diesem Grund wurde auch die Österreichische Zeitschrift für Soziologie (ÖZS) einer vergleichenden Analyse unterzogen.

\section{Religion in der ÖZS}

Die ÖZS eignet sich insbesondere für einen Vergleich, da die Soziologie zum einen eng mit der Politikwissenschaft verwandt ist. Zum anderen gilt ebenso für die ÖZS, dass es sich hierbei um die wissenschaftliche Fachzeitschrift im Bereich der Sozialforschung handelt. Sie wird seit 1976 von der Österreichischen Gesellschaft für Soziologie vierteljährlich im Verlag Springer VS herausgegeben. ${ }^{37}$ Insgesamt wurden 329 Artikel zwischen den Jahren 2001 und 2015 (Vol. 26, Issue 1, 2001 bis Volume 40, Issue 4, 2015) veröffentlicht, die hier untersucht werden. Dabei gilt für die ÖZS, dass sie im Unterschied zur ÖZP in erster Linie offene Hefte und keine spezifischen Themenhefte veröffentlicht. ${ }^{38}$

Im Falle der ÖZS fällt auf, dass der Durchschnittswert primär kodierter Artikel um mehr als die Hälfte niedriger liegt als bei der ÖZP. Mit 4,59 \% primär kodierter Artikel und 28,83 \% sekundär kodierter Artikel liegt der Wert in beiden Bereichen deutlich niedriger als im Falle der österreichischen politikwissenschaftlichen Zeitschrift.

\begin{tabular}{|l|l|l|l|}
\hline Zeitschriftenname & Artikelanzahl & $\begin{array}{l}\text { Primär kodiert } \\
(\mathbf{\%})\end{array}$ & $\begin{array}{l}\text { Sekundär kodiert } \\
(\mathbf{\%})\end{array}$ \\
\hline $\begin{array}{l}\text { Österreichische Zeit- } \\
\text { schrift } \\
\text { für Politikwissenschaft }\end{array}$ & 403 & 8,18 & 30,76 \\
\hline $\begin{array}{l}\text { Österreichische Zeit- } \\
\text { schrift } \\
\text { für Soziologie }\end{array}$ & 392 & 4,59 & 28,83 \\
\hline
\end{tabular}

Quelle: Darstellung des Autors

36 Vgl. Kettell 2012.

37 Vgl. Internetquellen: Kurze Geschichte der „Österreichischen Zeitschrift für Soziologie“.

38 Vgl. Internetquellen: Österreichische Zeitschrift für Soziologie. 
Ein Blick auf die Themenvielfalt verdeutlicht zudem einen weiteren Unterschied. Während das Thema Islam auch im Falle der ÖZS hoch rangiert, zeigt sich eine deutlichere Themenvielfalt im Vergleich zur ÖZP. Im Falle der ÖZS ist das Thema Islam nicht das dominanteste. Die Angabe von 16,66 \% (drei Artikel) ergibt sich, wenn ein Artikel, der Antisemitismus und Islamfeindlichkeit in vergleichender Perspektive zum Hauptthema hat, nicht zum Islam hinzugefügt wird. Dementsprechend würde das Hinzufügen dieses Artikels zum Islam einen Wert von 22,22 \% (vier Artikel) ergeben. Die Dominanz des Themenspektrums Wirtschaft ergibt sich vermutlich aus einer anhaltenden zentralen Bedeutung von Religion in der Erforschung von Gesellschaft und Ökonomie in den frühen soziologischen Schriften wie etwa Webers Arbeit zur protestantischen Ethik und dem "Geist“" des Kapitalismus. Im Gegensatz dazu erscheint der Begriff der Religion im Zusammenhang mit weiteren Themengebieten nur ein- bis zweimal, was auch daran liegen kann, dass die ÖZS im Gegensatz zum ÖZP kaum Themenhefte kennt. Dies führt im Falle der ÖZP dazu, dass bei Veröffentlichung eines Themenheftes mit Religionsbezug der prozentuelle Anteil an Artikel, welche Religion thematisieren, vergleichsweise hoch ausfällt.

\begin{tabular}{|l|l|l|l|l|}
\hline \multicolumn{2}{|l|}{ Primär kodierte Artikel: Vergleich zwischen ÖZS und ÖZP } \\
\hline & ÖZG & $\%$ & ÖZP & $\%$ \\
\hline & Thema & 27,77 & Islam/Muslime & 35,5 \\
\hline 1 & Wirtschaft & $22,22 /$ & Politische Theorie & 20,0 \\
\hline 2 & Islam/Muslime & 16,66 & & \\
\hline 3 & Gesellschaft & 11,11 & Cleavage-Theorie & 13,3 \\
\hline 4 & Politische Religion & 11,11 & Antisemitismus & 9,7 \\
\hline 5 & Politische Philosophie & 11,11 & Rechtspopulismus & 9,7 \\
\hline 6 & Religionszugehörigkeit & 5,55 & Religionszugehörigkeit & 6,5 \\
\hline 7 & Säkularität & 5,55 & - - & - - \\
\hline 8 & Antisemitismus & 5,55 & - - & - - \\
\hline 9 & Kolonialismus & 5,55 & - - - & - - \\
\hline
\end{tabular}

Quelle: Darstellung des Autors

Insgesamt kann gesagt werden, dass die Behandlung von Religion in der ÖZS die Grundlagen der Disziplin widerspiegelt. Zudem verweist der hohe Wert an 
Verbindung von Religion und Wirtschaft. Gleichzeitig ist der gleiche Trend zur Thematisierung von Islam/Muslime wie bei der ÖZP ersichtlich.

\section{Conclusio und weitere Reflexion}

Es zeigt in der Analyse der Thematisierung von Religion in der ÖZP, dass die Österreichische Politikwissenschaft im Vergleich zu den von Kettell erforschten Werten für die US-amerikanische Politikwissenschaft wie auch im Vergleich zur österreichischen soziologischen Zeitschrift ÖZS numerisch signifikant höher liegt. Die Thematisierung von Religion ist mit 8,18 \% aller Artikel vergleichsweise hoch. Verschiedene Erklärungen können hierfür herangezogen werden.

Im Vergleich zu den USA zeigt sich eine überdurchschnittliche Thematisierung von Religion in der ÖZP. Die Existenz von Themenheften im Falle der ÖZP legt nahe, dass damit automatisch gebündelt eine Vielzahl an Artikeln zu einem Themengebiet veröffentlicht wird, was sich im Gesamtbild entsprechend hoch niederschlägt. Die Kleinheit des Landes Österreich sowie die Nichtexistenz spezialisierter Organe wie etwa einer Sektion „Religion und Politik“ (inkl. eigenem Journal) in der Österreichischen Gesellschaft für Politikwissenschaft ermöglicht gleichzeitig eine höhere Präsenz der Thematisierung von Religion in der allgemeinen Politikwissenschaft, wie es in dieser Analyse der ÖZP reflektiert wird.

An dieser Stelle ein notwendiger Exkurs: Bei einzelnen AkteurInnen der Politikwissenschaft zeichnet sich ein steigendes Interesse an der Erforschung von Religion im Zusammenhang mit Politik ab. Die Wiener Politikwissenschaftlerin Sieglinde Rosenberger koordinierte von 2006 bis 2009 ein von der European Commission ( $6^{\text {th }}$ Framework Programme) gefördertes Forschungsprojekt zu Kopftuchpolitiken in Österreich. ${ }^{39}$ Von 2007 bis 2009 leitete sie das Forschungsprojekt Governing Difference. A challenge for New Democracies in Central and South Eastern European Countries, das religiöse, kulturelle und ethnische Differenz an der Schnittstelle zu Gender untersuchte. ${ }^{40}$ In dem 2008 gegründeten Forschungsprojekt The Politics of Inclusion \& Exclusion (INEX) setzt sie sich mit ihrem Forschungsteam mit religious governance auseinander. ${ }^{41}$ Die Innsbrucker Politikwissenschaftlerin Kristina Stoeckl beschäftigt sich seit Jahren mit dem Verhältnis von christlicher Orthodoxie und Politik. ${ }^{42}$ Die Salzburger Politikwissenschaftler Reinhard Heinisch und ich forschen in vergleichender Perspektive zu muslimischen Jugendbewegungen in Europa. ${ }^{43}$ Diese beispielhaften For-

39 Vgl. Internetquellen: VEIL. Values, Equality and Differences in Liberal Democracies.

40 Vgl. Internetquellen: Governing Difference.

41 Vgl. Internetquellen: INEX Politics of Inclusion and Exclusion.

42 Vgl. Internetquellen: Kristina Stoeckl.

43 Vgl. Internetquellen: Muslim Youth Movements in Europe. 
schungsinitiativen einzelner PolitikwissenschaftlerInnen reflektieren sich auch in neuen Einrichtungen. Im Unterschied zu den USA, wo mit Politics and Religion eine eigene Fachzeitschrift gegründet wurde, scheint sich in der österreichischen Politikwissenschaft eine neue Tendenz durchzusetzen, wonach explizit interdisziplinäre Formate sich der Thematisierung von Religion verschreiben. Dazu zählt etwa die begutachtete Reihe Religion and Transformation in Contemporary European Society, in der seit 2012 zehn Bände erschienen sind und die von den Mitgliedern der gleichnamigen Forschungsplattform an der Universität Wien, darunter unter anderem Sieglinde Rosenberger, herausgegeben wird. ${ }^{44}$ Während keine genuin politikwissenschaftlichen Einrichtungen zur Erforschung von Politik und Religion eingerichtet wurden, kann die Forschungsplattform Religion and Transformation an der Universität Wien im Bereich der Forschung als wichtige Einrichtung genannt werden, die auch die Politikwissenschaft umfasst. Weiters sind sieben Bände im 2010 gegründeten und seit 2015 einem Begutachtungsverfahren zugeführten Jahrbuch für Islamophobieforschung erschienen, die von mir herausgegeben werden. ${ }^{45}$ Diese beiden Fachzeitschriften reflektieren das mitunter steigende politikwissenschaftliche Interesse an einem interdisziplinären Zugang zur Erforschung von Religion. Dies mag zum einen der Einsicht geschuldet sein, dass die intellektuellen Grundlagen der Politikwissenschaft tatsächlich kein vollständiges Rüstzeug zur Verfügung stellen, um der Untersuchung des komplexen Gegenstands der Religion genügend Rechnung zu tragen, wie Wald und Wilcox meinen. Gleichzeitig mag ein Grund in der recht übersichtlichen politikwissenschaftlichen Landschaft eines so kleinen Landes wie Österreich liegen, die wenig Raum für weitere Ausdifferenzierungen der Politikwissenschaft innerhalb ihrer zentralen Institutionen bietet. Umgekehrt wird damit eine vermehrte Thematisierung von Religion durch einzelne PolitikwissenschaftlerInnen im Hauptorgan der österreichischen Politikwissenschaft, dem ÖZP, reflektiert.

Auch im Vergleich zur ÖZS zeigt sich, dass „Religion“ eine weitaus stärkere Relevanz in den Artikeln spielt. Dies kann institutionell erklärt werden. Es weist die Österreichische Gesellschaft für Soziologie in ihren 20 Sektionen keine einzige auf, die sich explizit mit Religion auseinandersetzt. ${ }^{46}$ Das ist ebenso der Fall für die acht eher klassisch orientierten Sektionen der Österreichischen Gesellschaft für Politikwissenschaft. ${ }^{47}$ Jedoch ist ein Gutteil der PolitikwissenschaftlerInnen im Bereich der Erforschung von Religion in der Forschungsplattform Religion and Transformation der Universität Wien organisiert und publiziert ihre Forschungen zu Religion mithilfe von Themenheften auch in der ÖZP.

Eine Gemeinsamkeit der beiden untersuchten Zeitschriften, der ÖZP wie der ÖZS, die sich mit den von Kettell untersuchten 20 US-amerikanischen Politik-

\footnotetext{
44 Vgl. Internetquellen: RaT.

45 Vgl. Internetquellen: Jahrbuch für Islamophobieforschung.

46 Vgl. Internetquellen: ÖGS.

47 Vgl. Internetquellen: ÖGPW.
} 
wissenschaftszeitschriften deckt, ist die hohe Beschäftigung mit dem Themengebiet Islam/Muslime. Damit könnte im Kontrast zu Wald / Wilcox nicht von einer Spezifität der ereignisgesteuerten Agenda der US-amerikanischen Politikwissenschaft alleine gesprochen werden. Schließlich gilt auch für die ÖZP, dass das Thema Islam wie auch im Falle der ÖZS sehr prominent oben rangiert.

Mit einem überdurchschnittlich hohen Anteil von 35,5\% aller Themen in Verbindung mit Religion (USA: 20 \%, ÖZS: 28,83 \%) ist gleichzeitig kritisch zu fragen, warum dieser Anteil so hoch ist. Gerade der Faktor Migration scheint in den Politikwissenschaften ein Impuls für eine vermehrte Beschäftigung mit Fragen von Religion gewesen zu sein. Das gilt insbesondere im Zusammenhang mit jenen Konflikten, in denen primär nicht-christliche eingewanderte Minderheiten um Anerkennung als ,gleichberechtigte Staatsbürger in einem durch erhebliche Asymmetrien geprägten religionspolitischen Komplex kämpfen“48.

So sehr die Hereinnahme von Religion im Zuge der Thematisierung von Migration auch einen gegebenen Anlass bietet, Religion tatsächlich in den Blick zu nehmen, so sehr ist dies meines Erachtens auch mit Risiken verbunden. Provokant möchte ich hier die Frage stellen, ob es erst eingewanderte MuslimInnen braucht, um das Feld der Religionspolitik abseits des Wiener Instituts für Rechtsphilosophie, Religions- und Kulturrecht zu entdecken. Wenn manche Kommentatoren die öffentliche obsessive Beschäftigung mit dem Islam als Projektion für die Auseinandersetzung mit historisch lange gewachsenen religiösen und etablierten religiösen Kräften in Österreich wie etwa der Katholischen Kirche brauchen, wäre auch in Richtung der Politikwissenschaft zu fragen: Wo ist die Auseinandersetzung mit Religion heute so wie vor 1979 abseits tagespolitischer Ereignisse? Abseits tagespolitischer Ereignisse: Wäre nicht der bleibende Einfluss des Cartellverbands in der Führungsriege der ÖVP - trotz des kurzen Intermezzos durch die Person Wolfgang Schüssel - ein gegebener Anlass für Forschungen im Bereich des Verhältnisses von Religion und Politik? Anders und allgemeiner gefragt: Übertünchen wir PolitikwissenschaftlerInnen nicht mit der Verquickung von Migration und Religion in der Forschung die Rolle von Religion in der Dominanzgesellschaft? Und vertieft dies nicht weiter einen blinden Fleck innerhalb der Disziplin?

Es bildet zwar keinen allgemeinen Konsens, aber zumindest für einen Teil der PolitikwissenschaftlerInnen besteht die Aufgabe dieser Disziplin auch in der Kritik und damit in der Intervention gegenüber bestehenden gesellschaftlichen und politischen Institutionen. ${ }^{49}$ Speziell die Thematisierung von Macht als zentraler politikwissenschaftlicher Kategorie würde es in einem katholisch geprägten

48 Willems / Minkenberg, S. 15. Der von Marian Burchardt und Ines Michalowski herausgegebene Sammelband „After Integration, Islam und Politik“ steht auch paradigmatisch für diese Verknüpfung von einheimisch gewordenen Minderheiten, die nach der Migration ihre Rechte beanspruchen.

49

Vgl. Albrecht 2013. 
Land, in dem die Katholische Kirche immer noch eine bedeutende gesellschaftliche Rolle spielt, nahelegen, sich stärker mit diesen Fragen auseinanderzusetzen.

So ist es begrüßenswert, wenn Politikwissenschaftlerinnen wie Permoser, Rosenberger und Stoeckl einen Beitrag vorgelegt haben, in dem sie die orthodoxe Kirche sowie die islamische Religionsgesellschaft als religiöse Akteurinnen untersuchen, ${ }^{50}$ da nicht die muslimische Gruppe als einzige in das Blickfeld genommen wird. Damit wird der Tendenz, die wir im Falle der Untersuchung von Kettell ebenso wie im Falle der Analyse der ÖZS und der ÖZP sehen konnten, nämlich ein Überhang an Beschäftigung mit dem Islam, ein breiterer Blick entgegengesetzt. Gleichzeitig wäre aber im Sinne der zuvor angesprochenen Diskussionspunkte die Frage aufzuwerfen, welche politikwissenschaftlichen Beiträge über die Katholische Kirche als politischer Akteurin existieren. Wenn Permoser in ihrer kürzlich erschienenen Analyse davon spricht, dass für österreichische Abgeordnete des Europaparlaments die eigene Religion privatisiert wird, während die Religion der „Anderen“ einer Politisierung unterläuft, ${ }^{51}$ könnte gefragt werden, ob nicht auch ähnliche Prozesse ablaufen, wenn heimische PolitikwissenschaftlerInnen sich mit der Religion der Immigrierten auseinandersetzen (Islam und christliche Orthodoxie) und der Religion der Mehrheit kaum Beachtung schenken. Insofern wäre weiter nachzufragen, inwiefern die Politikwissenschaft als Eliteprojekt nicht selbst auch dazu beiträgt, dass manche Aspekte - sei es bewusst oder unbewusst - weiterhin einen blinden Fleck bilden. Diese kritischen Anmerkungen scheinen mir an der Schnittstelle der von Wald und Wilcox angeführten Thesen des sozialen Hintergrunds der Proponenten der Politikwissenschaft sowie der These der ereignisgesteuerten Agenda der Disziplin von zentraler Bedeutung zu sein. Beide Thesen bedürfen einer weiteren Forschung für den Fall Österreich.

\section{Literaturverzeichnis}

Abromeit, Heidrun / Wewer, Göttrik: Die Kirchen und die Politik. Beiträge zu einem ungeklärten Verhältnis. Opladen 2013

Albrecht, Ulrich et al.: Was heißt und zu welchem Ende betreiben wir Politikwissenschaft? Kritik und Selbstkritik aus dem Berliner Otto-Suhr-Institut. Opladen 2013.

Bangstad, Sindre / Bunzl, Matti: ,,Anthropologists Are Talking About Islamophobia and Anti-Semitism in the New Europe“, in: Ethnos: Journal of Anthropology (75/2) 2010, S. 213-228.

Barbato, Mariano: „Auf der Suche nach einer postsäkularen ,Lingua Franca“? Die postsäkulare Gesellschaft von Jürgen Habermas am Beispiel der parlamentarischen Debatte zur Spätabtreibung“, in: Liedhegener, Antonius / Werkner, Ines-Jacqueline

50 Vgl. Mourão Permoser / Rosenberger / Stoeckl 2010.

51 Vgl. ebd. 
(Hg.): Religion zwischen Zivilgesellschaft und politischem System. Befunde - Positionen - Perspektiven. Wiesbaden 2011, S. 56-77.

Beyme, Klaus von: Religionsgemeinschaften, Zivilgesellschaft und Staat: Zum Verhältnis von Politik und Religion in Deutschland. Wiesbaden 2015.

Böckenförde, Ernst-Wolfgang: „Die Entstehung des Staates als Vorgang der Säkularisation“, in: Ders.: Recht, Staat, Freiheit. Studien zur Rechtsphilosophie, Staatstheorie und Verfassungsgeschichte. Frankfurt am Main 1992, S. 92-114.

Brocker, Manfred (Hg.): God bless America. Politik und Religion in den USA. Darmstadt 2013.

Bulliet, Richard et al.: The Princeton Encyclopedia of Islamic Political Thought. Ed. Gerhard Bowering. Princeton 2012.

Burchardt, Marian / Michalowski, Ines (eds.): After Integration. Islam, Conviviality and Contentious Politics in Europe (= Islam und Politik). Wiesbaden 2014.

Carty, Thomas J.: A Catholic in the White House? Religion, Politics, and John F. Kennedy's Presidential Campaign. New York / Hampshire 2004.

Casanova, José: Europas Angst vor der Religion. Berlin 2013.

Dachs, Herbert / Gerlich, Peter (Hg.): Politik in Österreich. Das Handbuch. Wien 2006.

Goethe, Johann Wolfgang: Faust. Der Tragödie erster Teil. WA 14, 1899.

Habermas, Jürgen / Ratzinger, Joseph: Dialektik der Säkularisierung. Freiburg / Basel / Wien 2005.

Hafez, Farid: „Islamophobe Weltverschwörungstheorien ... und wie Obama vom Muslim zum Muslimbruder wurde“, in: Journal für Psychologie (21/1) 2013, S. 1-22.

Jakubowski-Tiessen, Manfred (Hg.): Religion zwischen Kunst und Politik. Aspekte der Säkularisierung im 19. Jahrhundert. Göttingen 2004.

Kettell, Steven: „Has political science ignored religion?“, in: Political Science \& Politics (45/1) 2012, S. 93-100.

Lehmann, Hartmut (Hg.): Säkularisierung, Dechristianisierung, Rechristianisierung im neuzeitlichen Europa. Bilanz und Perpektiven der Forschung (= Veröffentlichungen des Max-Planck-Instituts für Geschichte 130). Göttingen 1997.

Ley, Michael / Neisser, Heinrich / Weiss, Gilbert (Hg.): Politische Religion? Politik, Religion und Anthropologie im Werk von Eric Voegelin (= Periagoge Studien). München 2003.

Morris, Aldon: „The Black Church in the Civil Rights Movement: the SCLC as the Decentralized, Radical Arm of the Black Church“, in: Smith, Christian (Hg.): Disruptive religion: The force of faith in social movement activism. New York / London 2014, S. 29-46.

Mourão Permoser, Julia / Rosenberger, Sieglinde / Stoeckl, Kristina: „Religious Organisations as Political Actors in the Context of Migration: Islam and Orthodoxy in Austria“, in: Journal of Ethnic and Migration Studies (36/9) 2010, S.1-19.

Ostendorf, Berndt: „Conspiracy Nation. Verschwörungstheorien und evangelikaler Fundamentalismus: Marion G. (Pat) Robertsons,Neue Weltordnung ““, in: Bielefeldt, Heiner / Heitmeyer, Wilhelm (Hg.): Politisierte Religion. Ursachen und Erscheinungsformen des modernen Fundamentalismus. Berlin 1998, S. 157-187.

Prainsack, Barbara: „Politik und Religion“, in: Dachs, Herbert / Gerlich, Peter (Hg.): Politik in Österreich. Das Handbuch. Wien 2006, S. 538-549. 
Rosenberger, Sieglinde / Sauer, Birgit (Hg.): Politics, religion and gender: Framing and regulating the veil. London / New York 2013.

Wald, Kenneth D. / Wilcox, Clyde: „Getting religion: Has political science rediscovered the faith factor?", in: American Political Science Review (100/4) 2006, S. 523-529.

Willems, Ulrich / Minkenberg, Michael: „Politik und Religion im Übergang. Tendenzen und Forschungsfragen am Beginn des 21. Jahrhunderts", in: Sonderheft Politische Vierteljahresschrift (33) 2003, S. 13-41.

\section{Internetquellen}

European Integration online Papers, verfügbar unter: http://eiop.or.at/eiop/vols_1997_ 2015.html [16.02.2016].

Governing Difference. A challenge for New Democracies in Central and South Eastern European Countries, verfügbar unter: http://typo3.univie.ac.at/index.php?id=17202 [16.02.2016].

INEX Politics of Inclusion and Exclusion: About Us, verfügbar unter: http://inex.univie. ac.at/ [16.02.2016].

Jahrbuch für Islamophobieforschung: Team, verfügbar unter: http://jahrbuch-islamo phobie.de/team/ [16.02.2016].

Kristina Stoeckl, verfügbar unter: http://www.kristinastoeckl.eu/links.html [16.02.2016].

Kurze Geschichte der „Österreichischen Zeitschrift für Soziologie“, verfügbar unter: http://agso.uni-graz.at/webarchiv/agsoe02/bestand/27_agsoe/27ges.htm [16.02.2016].

Mattersburger Kreis, Website, verfügbar unter: http://www.mattersburgerkreis.at/site/ de/publikationen/jep/alleausgabenartikel [12.10.2016].

Muslim Youth Movements in Europe, verfügbar unter: http://www.uni-salzburg.at/index. php $? \mathrm{id}=202291$ [16.02.2016].

ÖGPW: Die Sektionen der ÖGPW, verfügbar unter: http://www.oegpw.at/de/sektionen/ [16.02.2016].

ÖGS: Sektionen, verfügbar unter: http://oegs.ac.at/sektionen/ [16.02.2016].

Österreichische Gesellschaft für Politikwissenschaft, Website, verfügbar unter: http:// www.oegpw.at/de/ [16.02.2016].

Österreichische Gesellschaft für Politikwissenschaft: Call for Papers, verfügbar unter: http://www.oegpw.at/de/fileadmin/pdf/Tag_der_Politikwissenschaft_2014/Paper_Ab stracts/3_Polak.pdf [16.02.2016].

Österreichische Gesellschaft für Politikwissenschaft: Tagung: Europa und das Andere Konflikte um Geschlecht und Religion, verfügbar unter: http://www.oegpw.at/de/ak tuelles/archivmeldung/105-tagung-europa-und-das-andere-konflikte-um-geschlechtund-religion/ [16.02.2016].

Österreichische Zeitschrift für Soziologie, Vierteljahresschrift der Österreichischen Gesellschaft für Soziologie: All Volumes \& Issues, verfügbar unter: http://link.springer. com/journal/volumesAndIssues/11614 [16.02.2016].

ÖZP: Konzept, verfügbar unter: https://oezp.univie.ac.at/index.php/zfp/about/edito rialPolicies\#focusAndScope [16.02.2016]. 
Politics and Religion: Call for Proposals for New Editor(s), verfügbar unter: www.jour nals.cambridge.org/images/fileUpload/documents/RAP_Call_for_Editors_2015-16. pdf [16.02.2016].

Publikationsliste der Wiener Politikwissenschaftlerin Sieglinde Rosenberger, verfügbar unter: https://homepage.univie.ac.at/sieglinde.rosenberger/?page_id=12 [16.02.2016].

RaT: RaT-Reihe, verfügbar unter: http://www.religionandtransformation.at/publikatio nen/rat-reihe/ [16.02.2016].

Stoeckl, Kristina: Teaching, verfügbar unter: http://www.kristinastoeckl.eu/teaching. html [12.10.2016].

VEIL. Values, Equality and Differences in Liberal Democracies. Debates about Muslim Headscarves, verfügbar unter: http://inex.univie.ac.at/research/veil/ [16.02.2016]. 